$\overline{A E E T}$

ASOCIACIÓN ESPAÑOLA DE ECOLOGÍA TERRESTRE

\title{
El caracol acuático neozelandés del cieno (Potamopyrgus antipodarum): impactos ecológicos y distribución de esta especie exótica en la península ibérica
}

\author{
A. Alonso ${ }^{1, *}$, P. Castro-Díez ${ }^{1}$ \\ (1) Departamento de Ciencias de la Vida, Unidad Docente de Ecología, Universidad de Alcalá, Crta. N-II Km 33,6 Campus Universitario, Edificio Biología, \\ 28805 Alcalá de Henares, Madrid, España. \\ *Autor de correspondencia: A. Alonso [aafernandez1976@yahoo.es]
}

> Recibido el 07 de julio de 2014 - Aceptado el 08 de octubre de 2014

\begin{abstract}
Alonso, A., Castro-Diéz, P. 2015. El caracol acuático neozelandés del cieno (Potamopyrgus antipodarum): impactos ecológicos y distribución de esta especie exótica en la península ibérica. Ecosistemas 24(1): 52-58. Doi.: 10.7818/ECOS.2015.24-1.09

Las especies exóticas invasoras constituyen una de las principales causas de pérdida de biodiversidad. Debido a la complejidad del proceso de invasión, algunas especies muestran comportamiento invasor en unos ecosistemas pero no en otros. Una especie con un comportamiento ambiguo respecto a su carácter invasor es el caracol del cieno de Nueva Zelanda (Potamopyrgus antipodarum). La inclusión de esta especie en el reciente catálogo español de especies exóticas invasoras obliga a establecer su seguimiento e incrementar el conocimiento sobre su distribución en España. En el presente estudio se ha revisado la distribución de la especie en la península ibérica y los impactos ecológicos que ocasiona. En España la especie se ha citado en 30 de las 46 provincias, mientras que en Portugal aparece en 6 de 18. Muchas de las provincias afectadas son costeras, especialmente las que cuentan con las citas más antiguas. Respecto a los impactos más destacables que genera destaca su capacidad para cambiar la estructura de las comunidades de invertebrados a las que afecta, aunque en determinados casos no se han descrito tales cambios. La revisión de trabajos muestra una carencia de estudios que indiquen los mecanismos de dispersión de la especie entre distintas cuencas en la Península Ibérica, aspecto muy importante para evitar la expansión de la especie en los ecosistemas acuáticos peninsulares.
\end{abstract}

Palabras clave: especies invasoras; ecosistemas acuáticos; invertebrados acuáticos; moluscos

Alonso, A., Castro-Diéz, P. 2015. The New Zealand mudsnail (Potamopyrgus antipodarum): ecological impacts and distribution of this exotic species in the Iberian Peninsula. Ecosistemas 24(1): 52-58. Doi.: 10.7818/ECOS.2015.24-1.09

Biological invasions are one of the main causes of biodiversity lost. Given the complexity of the invasion processes, some species have shown invasive behaviour in some ecosystems but not in others. A species with an ambiguous invasive behaviour is the New Zealand mudsnail (Potamopyrgus antipodarum). This species has been recently included as an invasive exotic species in Spanish law, which makes necessary the management and the knowledge of its distribution in Spain. In this study we have reviewed the distribution and ecological impacts of this species in the Iberian Peninsula. In Spain this species has been cited in 30 out of the 46 provinces, whereas in Portugal it has been cited in 6 out of 18 . Most of the affected provinces are in the coast, especially in the provinces with the oldest quotes. Among the most important impacts caused by this species are its ability to change the structure of the invaded invertebrate communities. However, such impacts have not been reported in some case studies. The present review shows a lack of studies on the dispersal mechanisms of mudsnail throughout the Iberian Peninsula. This is an essential point to prevent the spread of this species throughout the aquatic ecosystems of the Iberian Peninsula.

Key words: invasive species; aquatic ecosystems; aquatic invertebrates; molluscs

\section{Introducción}

Las especies exóticas invasoras son una de las principales causas de pérdida de biodiversidad a escala global (Gherardi 2007; Netwing 2007). Muchas actividades humanas, como el transporte de mercancías, han aumentado las oportunidades de muchas especies para ampliar su área de distribución. En ciertas ocasiones, algunas de ellas se convierten en especies exóticas invasoras en las nuevas áreas al expandirse y causar un impacto en la estructura y funcionamiento de los ecosistemas invadidos (Gherardi 2007; Netwing 2007).

El proceso por el cual una especie exótica se convierte en invasora es complejo e implica la superación de filtros sucesivos (Castro-Díez et al. 2004). Esto hace que solamente una pequeña parte de las especies exóticas se conviertan en invasoras (Kolar y
Lodge 2001). Hay muchos factores que influyen en las probabilidades de éxito a lo largo de este proceso, tal como la idoneidad de las condiciones ambientales en el ecosistema receptor, las características de la propia especie (reproducción exitosa y rápida, tolerancia amplia a factores ambientales, etc.) o la frecuencia e intensidad con que se introduce la especie. Debido a la complejidad del proceso de invasión, algunas especies presentan comportamientos invasores en determinados ambientes (i.e. causan algún cambio importante sobre las propiedades del ecosistema receptor), mientras que en otros no pasan de ser una especie más dentro del ecosistema (la especie se naturaliza sin causar cambios considerables en el ecosistema receptor) (Kumschick et al. 2012; Castro-Díez et al. 2014). Las causas para que algunas especies se comporten de este modo no son del todo conocidas. En los ecosistemas acuáticos hay numerosos ejemplos de especies exóticas invasoras con 
un claro impacto en todos los ecosistemas invadidos, tal como el mejillón cebra (Dreissena polymorpha), la gamba asesina (Dikerogammarus villosus), el caracol manzana (Pomacea canaliculata) o el alga asesina (Caulerpa taxifolia) (Lowe et al. 2000; Baur y Schmidlin 2007). Sin embargo, calificar a algunas especies como exóticas invasoras no es tarea fácil, ya que su impacto varía dependiendo de las condiciones ambientales del medio, la presión biótica, el tipo de ecosistema, siendo el impacto en ocasiones nulo (Lowe et al. 2000; Baur y Schmidlin 2007).

Una especie con un comportamiento ambiguo respecto a su carácter invasor es el caracol acuático neozelandés del cieno (Potamopyrgus antipodarum). Éste molusco prosobranquio, de la familia Hydrobiidae, cuenta con una concha de entre 6 y $7 \mathrm{~mm}$ de longitud en las zonas invadidas, aunque en su región nativa puede llegar a los $12 \mathrm{~mm}$ (Winterbourn 1970). Es una especie tolerante a la desecación, ya que durante los periodos secos o más calientes se entierra en el sedimento, lo que le permite sobrevivir más de un día fuera del agua, con supervivencias del $50 \%$, de ahí su nombre común "caracol del cieno" o mudsnail en inglés (Alonso y CastroDíez 2012a, b; Duft et al. 2003). Una de las características más peculiares que presenta esta especie es su reproducción partenogenética en las zonas invadidas, mientras que en su área nativa predomina la reproducción sexual (Alonso y Castro-Díez 2012a). Generalmente, este tipo de reproducción permite a algunas especies exóticas tener una mayor probabilidad de éxito invasor, como se ha descrito en algunas especies de cangrejos o de lombrices de tierra (Terhivuo y Saura 2006; Jones et al. 2009). Este caracol alcanza la madurez sexual relativamente rápido, considerándose adultos con una longitud de concha de 3-3.5 mm (Richards 2002). Se trata de una especie ovovivípara, es decir, porta los huevos en un receptáculo de cría donde eclosionan y salen al exterior a través de la apertura de la concha, de tal forma que nacen pequeños caracoles transparentes que con los días adquirirán el color oscuro de su concha (Jokela et al. 1997). La fecundidad de esta especie es muy elevada, en un año un adulto puede llegar a reproducirse en hasta seis ocasiones, pudiendo tener 230 caracoles de media por adulto y año, lo que facilita su éxito invasor en muchos ecosistemas (Alonso y Castro-Díez 2012a).

La dieta de $P$. antipodarum es muy amplia, incluyendo principalmente microorganismos característicos del perifiton (algas y bacterias), y hongos que se desarrollan sobre la materia orgánica en descomposición (Dorgelo y Leonards 2001; Jensen et al. 2001; Alonso y Camargo 2003; Duft et al. 2003; Alonso y Castro-Díez 2008; 2012a). Por lo tanto, se trata de una especie muy generalista capaz de utilizar una amplia variedad de recursos tróficos. Asimismo esta especie puede usar un amplio margen de hábitats, pudiendo vivir tanto en aguas dulces como salobres (Winterbourn 1973), en zonas con sedimento fino acumulado, entre las plantas acuáticas, sustratos duros o arena, también presenta un amplio margen de tolerancia a diferentes condiciones hidráulicas, aunque las velocidades de corriente muy elevadas no le favorecen (Holomuzki y Biggs 2007). Éste amplio margen de tolerancia frente a distintos factores ambientales da una idea del amplio rango de ecosistemas que potencialmente puede ocupar esta especie.

Estudios recientes muestran la alta capacidad de $P$. antipodarum para emplear diferentes formas de dispersión tanto pasivas (en las patas o el plumaje de aves acuáticas, en el barro que se adhiere a embarcaciones o vehículos, anclado al pelo de animales, en el estómago de peces) como activas (por medio de su propio desplazamiento aguas arriba en ríos) (Alonso y Castro-Díez 2008; 2012a; Sepulveda y Marczak 2012). Su elevada capacidad de dispersión hace que $P$. antipodarum se propague fácilmente una vez ha colonizado un medio. Por ejemplo, se ha comprobado que $P$. antipodarum tolera varias horas en el intestino de peces y puede sobrevivir tras ser expulsados en sus heces (Vinson y Baker 2008; Alonso y Castro-Díez 2008). El movimiento de agua entre distintas masas de agua localizadas en distintas cuencas mediante transvases también podría fomentar la dispersión de esta especie exótica, como ya se ha visto para otras especies exóticas (Rahel 2007).
En cuanto a los impactos causados por esta especie sobre la estructura y funcionalidad de los ecosistemas acuáticos invadidos a lo largo del mundo, se ha descrito que ocasiona grandes cambios en la comunidad de moluscos de diferentes ecosistemas acuáticos (Gèrard et al. 2003; Lewin y Smolinski 2006), cambia los ciclos de $\mathrm{N}$ y $\mathrm{C}$, y la producción secundaria en algunos ríos (Hall et al. 2003, 2006). Esta especie puede alcanzar elevadas densidades en alguno de los ecosistemas que invade, aspecto que causa una profunda modificación de la estructura de la comunidad de invertebrados, ya que se han descrito densidades de hasta 500000 caracoles por $\mathrm{m}^{2}$ (Hall et al. 2003). Además puede alcanzar producciones secundarias muy elevadas, con los valores más altos descritos para invertebrados fluviales (Hall et al. 2003). También se ha descrito como esta especie puede reducir el crecimiento de especies nativas de caracoles por competencia trófica (Riley et al. 2008). Finalmente esta especie causa una reducción del crecimiento en la trucha arcoíris (Oncorhynchus mykiss) cuando es alimentada principalmente con P. antipodarum (Vinson y Baker 2008).

En España esta especie ha sido recientemente considerada como "invasora" en el Real Decreto 630/2013, de 2 de agosto, por el que se regula el catálogo español de especies exóticas invasoras. En dicho Real Decreto P. antipodarum ha sido incluida como especie "exótica invasora", lo que hace obligatorio un seguimiento y estudio en profundidad de su distribución en España. No obstante, la información disponible sobre la distribución de esta especie en España es escasa y dispersa. Lo mismo sucede con sus impactos ecológicos y económicos en los ecosistemas acuáticos que invade. El objetivo de la presente revisión es determinar la distribución de la especie en los ecosistemas acuáticos de la península ibérica (incluyendo los territorios peninsulares de España y Portugal) y sus impactos ecológicos en los mismos, a través de la revisión de la información científica publicada al respecto. Con ello se pretende mejorar el estado de conocimiento de la distribución de la especie y de las necesidades futuras de investigación.

\section{Material y Métodos}

Se realizó una búsqueda bibliográfica empleando buscadores científicos (ISI WEB of Knowledge, Scirus, Google Scholar) sin acotar fechas y utilizando palabras clave relacionadas con la ecología acuática (aquatic ecosystem, river, stream, lake, etc. y sus equivalentes en castellano), los impactos (impact, exotic species, invasive species, ecological impact, etc. y sus equivalentes en castellano) y la especie (Potamopyrgus antipodarum o su sinónimo Potamopyrgus jenkinsi, mud snail, mudsnail). Se seleccionaron aquellos artículos que por su título y/o resumen fuesen relevantes para el objetivo del trabajo. Se ha utilizado aquella información que tiene un filtro de revisión por pares y/o editorial. En cada uno de los artículos se buscó información sobre la distribución de la especie en las diferentes provincias y regiones de España y Portugal, datos sobre su abundancia, preferencias de hábitat, y sobre los impactos ecológicos de la especie en los ecosistemas acuáticos invadidos. Adicionalmente, se localizaron las citas de la presencia de $P$. antipodarum en la península ibérica en la base de datos GBIF (Global Biodiversity Information Facility), para compararlos con los datos localizados en las diferentes referencias bibliográficas revisadas.

\section{Resultados}

La Figura 1 muestra las provincias españolas y portuguesas en donde se ha citado la presencia de esta especie en al menos una localización (30 de 46 en España, y 6 de 18 en Portugal) según las referencias bibliográficas revisadas en el presente estudio. En las localizaciones de la especie según GBIF encontramos una provincia (La Rioja) con presencia de $P$. antipodarum y que no ha podido ser encontrada en la revisión bibliográfica. No obstante, y a la vista de la Figura 1, dicha base de datos subestima la presencia de $P$. antipodarum en la península ibérica, relegándola principal- 
Tabla 1. Lugares y provincias de España y Portugal donde se ha citado la presencia de Potamopyrgus antipodarum, en al menos una ocasión. Para cada lugar se indica el tipo de ecosistema, sus posibles impactos, las características ecológicas y la abundancia de la especie (individuos por $m^{2}$ ) cuando está disponible.

Table 1. Locations and provinces in Spain and Portugal where the presence of Potamopyrgus antipodarum has been reported at least once. For each location we report the type of invaded ecosystem, the likely impacts and ecological properties, and the (individuals per $m^{2}$ ) when available.

\section{País Provincias Lugar Ecosistema Impactos, características ecológicas, Abundancia Referencia comunidad nativa $\quad$ (indvs $/ \mathrm{m}^{2}$ )}

España Cantabria Cuenca del Pas Ríos

\begin{tabular}{lll} 
Cantabria & Cuenca del Pas & Ríos \\
\hline Cantabria & $\begin{array}{l}\text { Río Deva y Río } \\
\text { Miera }\end{array}$ & Ríos \\
\hline Huelva & $\begin{array}{l}\text { Río Odiel y Río } \\
\text { Tinto }\end{array}$ & Ríos \\
\hline Cádiz & Laguna Tarelo & Laguna \\
\hline Málaga & Río Genal & Rezumadero \\
\hline Navarra & Río Arga & Río
\end{tabular}

\begin{tabular}{|c|c|c|c|c|c|}
\hline Navarra & - & $\begin{array}{l}\text { Ríos, arroyos y } \\
\text { canales de riego }\end{array}$ & $\begin{array}{l}\text { Muy común en hábitats lóticos de la provincia de } \\
\text { Navarra }\end{array}$ & 30000 & $\begin{array}{l}\text { Larraz y Equisoain JJ } \\
\text { (1993) }\end{array}$ \\
\hline $\begin{array}{l}\text { Cáceres y } \\
\text { Badajoz }\end{array}$ & $\begin{array}{l}\text { Canal de } \\
\text { Orellana }\end{array}$ & Canal & Elevadas densidades respecto a otros moluscos & - & $\begin{array}{l}\text { Bech-Taberner y } \\
\text { Altamiras-Roset (2003) }\end{array}$ \\
\hline Madrid & $\begin{array}{l}\text { Muy extendido } \\
\text { por la provincia }\end{array}$ & $\begin{array}{l}\text { Varios ríos, } \\
\text { arroyos, lagunas, } \\
\text { barrancos } \\
\text { húmedos, } \\
\text { acequias y } \\
\text { canales de riego }\end{array}$ & - & - & Soler et al (2006) \\
\hline Guipúzcoa & $\begin{array}{l}\text { Ríos Bidasoa, } \\
\text { Oiarztun, } \\
\text { Urumea, Oria, } \\
\text { Urola }\end{array}$ & Ríos & Bajas densidades $(<500)$ & - & Arluziaga (2002) \\
\hline Murcia & $\begin{array}{l}\text { Cuenca del } \\
\text { Segura }\end{array}$ & Ríos & $\begin{array}{l}\text { Dentro de los moluscos fue la especie que } \\
\text { presentó mayor afinidad por tramos fluviales } \\
\text { sometidos a intenso uso agrícola }\end{array}$ & - & $\begin{array}{l}\text { Mellado-Díaz et al } \\
(2008)\end{array}$ \\
\hline Barcelona & $\begin{array}{l}\text { Arroyo La } \\
\text { Tordera }\end{array}$ & Arroyo & $\begin{array}{l}\text { Aparece aguas arriba y aguas abajo de un } \\
\text { efluente de depuradora } \\
\text { Tiende a ser más abundante en invierno. }\end{array}$ & $12-55$ & $\begin{array}{l}\text { Ortiz et al. (2005) } \\
\text { Ortiz y Puig (2007) }\end{array}$ \\
\hline Barcelona & Arroyo Vallvidrera & Arroyo & $\begin{array}{l}\text { Su mayor abundancia se da en zonas con } \\
\text { contaminación intermedia por materia orgánica } \\
\text { No afecta a la cantidad de clorofila a } \\
\text { Es dominante en todos los microhábitats fluviales } \\
\text { Las altas velocidades de corriente no le favorecen } \\
\text { Interacciona negativamente con las familias } \\
\text { Erpobdellidae y Chironomidae }\end{array}$ & $0-35000$ & Múrria et al. 2008 \\
\hline Guadalajara & Río Henares & Río & $\begin{array}{l}\text { Aparece en tramos de cabecera degradados } \\
\text { (contaminación orgánica y/o rectificación de } \\
\text { cauces) }\end{array}$ & - & Alonso (2005) \\
\hline
\end{tabular}

Más abundante hacia los tramos bajos Posible buen re-colonizador tras episodios de incrementos de caudal

Junto con los anfípodos forman el $90 \%$ de la comunidad de macroinvertebrados

Su abundancia aumenta aguas abajo de efluentes de depuradora

Prefiere zonas con poca corriente

Presente en los tramos de cabecera mejor conservados

Laguna con pobre calidad ecológica

Presente en tramos medio-altos hasta tramos bajos

Alta tolerancia a la contaminación orgánica

Especie más abundante de la comunidad
1376-13 072 Álvarez-Cabria et al. (2011a)

Álvarez-Cabria et al. (2010)

Álvarez-Cabria et al. (2011b)

- $\quad$ Pérez-Quintero (2011)

- $\quad$ Serrano et al. (2004)

- $\quad$ Gavira Romero et al. (2009)

- $\quad$ Jiménez y Larraz (1986)

(n)




\section{País Provincias Lugar Ecosistema Impactos, características ecológicas, Abundancia Referencia comunidad nativa \\ (indvs $/ \mathrm{m}^{2}$ )}

\begin{tabular}{|c|c|c|c|c|c|c|}
\hline \multirow[t]{9}{*}{ España } & Guadalajara & Río Cañamares & Río & $\begin{array}{l}\text { Llega a suponer el } 74 \% \text { de promedio anual de la } \\
\text { comunidad de macroinvertebrados }\end{array}$ & - & $\begin{array}{l}\text { de la Puente y } \\
\text { Camargo (2006) }\end{array}$ \\
\hline & Albacete & Sierra de Alcaraz & Ríos y arroyos & - & - & $\begin{array}{l}\text { Martínez-Ortí et al. } \\
(2004)\end{array}$ \\
\hline & Córdoba & $\begin{array}{l}\text { Dehesa de Sierra } \\
\text { Morena }\end{array}$ & Aguadulce & - & - & Pérez-Quintero (2009) \\
\hline & Cuenca & $\begin{array}{l}\text { Varias } \\
\text { localizaciones }\end{array}$ & Arroyos & - & - & Werner et al 1994 \\
\hline & $\begin{array}{l}\text { Lérida, } \\
\text { Huesca, } \\
\text { Zaragoza, } \\
\text { Navarra, } \\
\text { Álava, Teruel }\end{array}$ & $\begin{array}{l}\text { Cuenca del río } \\
\text { Ebro }\end{array}$ & Ríos & Ampliamente distribuida por la cuenca & - & Oscoz et al. (2010) \\
\hline & Granada & $\begin{array}{l}\text { Varias } \\
\text { localizaciones }\end{array}$ & $\begin{array}{l}\text { Arroyos, canales, } \\
\text { fuentes }\end{array}$ & $\begin{array}{l}\text { Distribuida por las costas españolas, sin citas } \\
\text { para el interior de la península ibérica }\end{array}$ & - & Ibáñez y Alonso (1977) \\
\hline & $\begin{array}{l}\text { Barcelona, } \\
\text { Gerona, } \\
\text { Guipúzcoa, } \\
\text { Vizcaya, } \\
\text { Cantabria, } \\
\text { Asturias, } \\
\text { La Coruña, } \\
\text { Alicante, } \\
\text { Lérida, } \\
\text { Valencia, } \\
\text { Huesca, } \\
\text { Castellón de } \\
\text { la Plana, } \\
\text { Tarragona }\end{array}$ & $\begin{array}{l}\text { Varias } \\
\text { localizaciones }\end{array}$ & $\begin{array}{l}\text { Varios } \\
\text { ecosistemas }\end{array}$ & - & - & Ibáñez y Alonso (1977) \\
\hline & Murcia & $\begin{array}{l}\text { Cuenca del río } \\
\text { Segura }\end{array}$ & $\begin{array}{l}\text { Aguas de poca } \\
\text { corriente Tramos } \\
\text { bajos de arroyos }\end{array}$ & - & - & Mellado-Díaz (2005) \\
\hline & Pontevedra & Río Miño & $\begin{array}{l}\text { Tramo bajo del } \\
\text { río Miño }\end{array}$ & $\begin{array}{l}\text { Gran parte del tiempo fuera del agua en zonas de } \\
\text { fango y humedad }\end{array}$ & 98300 & Rolán (2004) \\
\hline \multirow[t]{7}{*}{ Portugal } & Setubal & Santo André & Laguna costera & Asociado a altos niveles de salinidad & - & Correia et al. (2012) \\
\hline & Faro & $\begin{array}{l}\text { Ribeira de } \\
\text { Odelouca, } \\
\text { ribeira do Arade } \\
\text { em Silves, } \\
\text { Ribeira de } \\
\text { Enxerim }\end{array}$ & & - & - & Simoes (1988) \\
\hline & Santarém & - & Canal de riego & Grandes abundancias & - & Simoes (1988) \\
\hline & Aveiro & - & - & - & - & Simoes (1988) \\
\hline & Leiria & - & - & - & - & Simoes (1988) \\
\hline & $\begin{array}{l}\text { Viana do } \\
\text { Castelo }\end{array}$ & $\begin{array}{l}\text { Estuario del Río } \\
\text { Miño }\end{array}$ & $\begin{array}{l}\text { Estuario (zona } \\
\text { de agua dulce) }\end{array}$ & - & $0-2044$ & $\begin{array}{l}\text { Sousa et al. (2007) } \\
(2005)\end{array}$ \\
\hline & Setúbal & Lago de Melides & $\begin{array}{l}\text { Lago costero } \\
\text { salobre }\end{array}$ & $\begin{array}{l}\text { Poblaciones constantes a lo largo del año } \\
\text { independientemente de los cambios de salinidad }\end{array}$ & - & Costa et al (2003) \\
\hline
\end{tabular}




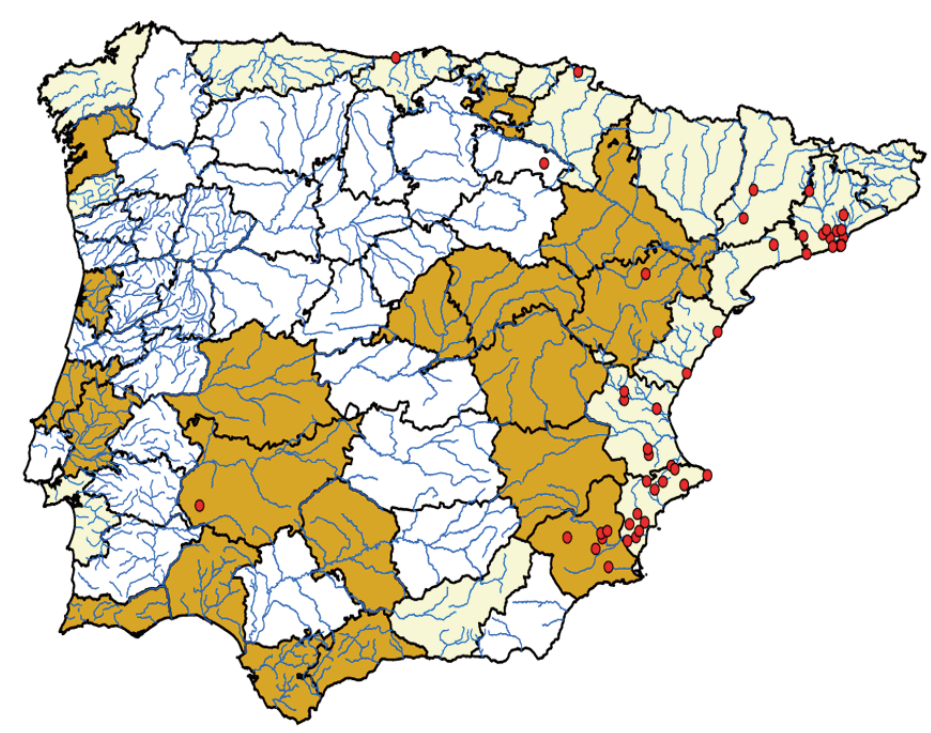

Figura 1. Mapa de la península ibérica con las provincias españolas y portuguesas donde hay al menos una cita de la presencia de Potamopyrgus antipodarum según la bibliografía revisada en el presente trabajo. En color amarillo se indican las provincias con citas anteriores a 1990, y en color naranja las citas posteriores. Los puntos rojos corresponden a citas encontradas en GBIF (Global Biodiversity Information Facility.

Figure 1. Map of Iberian Peninsula showing the Spanish and Portuguese provinces where the presence of Potamopyrgus antipodarum has been reported at least once in the revised references of the present study. Yellow color shows provinces with the quotes before 1990, orange color shows later quotes. Red points show quotes that have been reported in GBIF (Global Biodiversity Information Facility.

mente al levante español. En la Tabla 1 se recoge la información detallada de las localizaciones de la especie en la península ibérica, el tipo de ecosistema en el que habita y las características ecológicas o sus posibles impactos en el medio. Como se puede apreciar, $P$. antipodarum se encuentra en numerosas localizaciones a lo largo de las zonas costeras, y también en algunas provincias del interior de España, $P$. antipodarum habita ecosistemas acuáticos con características ambientales muy diferentes, desde ecosistemas de agua dulce, como arroyos, ríos, lagunas y fuentes, hasta ecosistemas costeros y salobres. Esto da una idea del amplio margen de ambientes en los que la especie puede vivir y mantener poblaciones estables. Incluso se ha encontrado a la especie habitando gran parte del tiempo fuera del agua en zonas de fango y humedad elevada (Rolán 2004). A la vista de los ecosistemas donde se ha encontrado la especie, son los de agua dulce -y dentro de estos los fluviales- los más frecuentes. En general se observa una carencia de datos respecto a las abundancias de la especie en las diferentes localizaciones (con solamente seis estudios que proporcionan datos de la abundancia de la especie). En estos estudios se muestra un amplio margen de abundancias desde unas pocas decenas de individuos por $\mathrm{m}^{2}$ hasta cerca de 100000 (Rolán 2004; Ortiz et al. 2005; Ortíz y Puig 2007). Respecto al número de estudios que cuantifican los impactos ecológicos de la especie en el ecosistema receptor, la información revisada muestra que este apartado no ha sido muy estudiado. No obstante, parece que la degradación ambiental (cuencas agrícolas, vertidos de materia orgánica, etc.) favorece a la especie (Jiménez y Larraz 1986; Mellado-Díaz et al. 2008; Múrria et al. 2008; Alonso 2005; Álvarez-Cabria et al. 2011b). Además, la baja velocidad de corriente parece que también le es favorable, evitando lugares con corrientes elevadas (Álvarez-Cabria et al. 2011b), aspecto que se ha comprobado en otros estudios de forma experimental y en campo (Holomuzki y Biggs 2007). No obstante, también se han encontrado casos en los que la especie se ve relegada a zonas sin contaminar en ecosistemas fluviales (Pérez-Quintero 2011), lo cual pudo deberse a la severa contaminación de tramos fluviales más bajos.

\section{Discusión}

Algunos de los primeros estudios sobre la distribución de esta especie en España (Ibáñez y Alonso 1977) y Portugal (Simoes 1988 ) ya indicaron su presencia en numerosas localizaciones de la península ibérica. Ibáñez y Alonso (1977) indicaron que la mayoría de las citas de $P$. antipodarum en España se daban en las provincias costeras cantábricas y mediterráneas, con pocas citas en regiones del interior. Algo similar sucede con la revisión de Simoes (1988) en Portugal, donde las citas se localizaban en la costa o en zonas próximas a la misma. Actualmente este patrón ha cambiado, ya que muchas de las citas más recientes indican su presencia en zonas del interior peninsular (Tabla 1, Fig. 1), lo cual puede indicar una tendencia expansiva de la especie, tal y como ha ocurrido en otras zonas geográficas, como la costa oeste de Estados Unidos y Australia (Hosea y Finlayson 2005; Loo et al. 2007). La localización costera de $P$. antipodarum durante los primeros estudios en la península ibérica probablemente indica que la vía de llegada de $P$. antipodarum pudo haber sido a través de aguas de lastre de embarcaciones, aunque algunos autores apuntan que a partir del primer punto de entrada de la especie a Europa (situado en el estuario del Támesis, donde llegó a través de barriles de agua en el S.XIX) la dispersión se realizó principalmente por medio de aves acuáticas (Ibáñez y Alonso 1977). No hay que descartar tampoco la posibilidad de una carencia de estudios en zonas del interior de la Península durante aquellos años. En cualquier caso, en vista de la distribución actual de la especie con respecto a la década de los $70-80$, podemos indicar una ampliación de la distribución hacia ecosistemas acuáticos del interior (ríos, lagos y canales). La dispersión entre cuencas o entre tramos de un mismo río a través de aves, aparejos de pesca, embarcaciones y tracto digestivo de peces se han citado en otros países como mecanismos de dispersión de la especie (Zaranko et al. 1997; Gangloff 1998; Hosea y Finlayson 2005; Alonso y CastroDíez 2008). Aunque nuestros ecosistemas carecen de estudios sobre los principales medios de dispersión, se deberían implementar medidas basadas en los conocimientos adquiridos en otras regiones (por ejemplo desinfección de cualquier material empleado en zonas con presencia de la especie) para evitar su dispersión (Hosea y Finlayso 2005). Cabe destacar que aunque la especie está presente en numerosos sitios de la península ibérica, las bases de datos disponibles a escala global o europea prácticamente relegan a la especie a unas pocas localizaciones, careciendo de información completa. En el caso del Global Biodiversity Information Facility (GBIF) la especie no aparece en Portugal, y en España lo hace para un total de 76 localizaciones, la mayoría de ellas citadas en provincias del este (Barcelona, Lérida, Tarragona, Teruel, Castellón, Valencia, Alicante, Murcia) y con unas pocas localizaciones en provincias del norte (La Rioja, Cantabria y Navarra) y del oeste (Badajoz). En el caso de la Global Invasive Species Databese (GISD) aparece como establecida para España y sin información para Portugal, indicando la presencia de la especie en Madrid, Guadalajara y Cataluña. Y finalmente en el caso de la Delivering Alien Invasive Species Inventories for Europe (DAISIE) no aparece ni en España ni en Portugal. Lo anterior denota la necesidad de contribuir a un mejor conocimiento de la distribución de la especie en la península ibérica sin el cual es muy difícil tomar medidas adecuadas para el control de la especie.

Aunque son relativamente numerosos los estudios que citan la presencia de la especie en la península ibérica, es muy escasa la literatura científica que trata de evaluar los efectos ecológicos de este gasterópodo sobre las comunidades acuáticas de los ecosistemas peninsulares. Recientemente se han revisado los impactos de esta especie en diferentes ecosistemas a lo largo del mundo (Alonso y Castro-Díez 2012a). En el caso de la península ibérica se puede destacar el estudio de Murria et al. (2008), que analiza los impactos ecológicos de la especie sobre la comunidad de macroinvertebrados bentónicos de un arroyo de agua dulce en la provincia de Barcelona. En dicho estudio no se apreciaron impactos claros sobre la estructura de la comunidad receptora ni 
sobre la producción primaria, siendo este gasterópodo más abundante en zonas con un nivel de contaminación orgánica no muy alta. Por el contrario el estudio de de la Puente y Camargo (2006) indicó que la especie es dominante en la comunidad de macroinvertebrados de un río del centro de la Península, representando el $74 \%$ del promedio anual de la abundancia de dicha comunidad. Este último estudio coincide con estudios realizados en Estados Unidos (Hall et al. 2003; 2006), donde la especie se hace dominante en la comunidad de macroinvertebrados bentónicos. Por otro lado algunos autores han apuntado que las especies exóticas se pueden ver favorecidas por la contaminación, ya que ésta supone un pulso de recursos que pueden ser utilizados por especies recién llegadas al ecosistema (Crooks et al. 2011; Svobodova et al. 2012). En el caso de España y para $P$. antipodarum, varios estudios indican cómo la especie tiene afinidad por zonas contaminadas con materia orgánica y/o eutrofizadas (Jiménez y Larraz 1986; Alonso 2005; Mellado-Díaz et al. 2008; Múrria et al. 2008; Álvarez-Cabria et al. 2011b). En la revisión de Alonso y CastroDíez (2008) sobre las propiedades biológicas que podían explicar el éxito invasor de la especie, se apuntó que una de las posibles causas sería su elevada tolerancia a factores abióticos, entre ellos la contaminación por materia orgánica y la eutrofización de las aguas. De hecho, parte de esta tolerancia ha sido demostrada a través de estudios de laboratorio, en donde se comprobó la alta resistencia de la especie al amoniaco, nitrito y nitrato (Alonso y Camargo 2003, 2004; Alonso 2005). No obstante, en un estudio desarrollado en los ríos Odiel y Tinto (provincia de Huelva) por Pérez-Quintero (2011) se muestra cómo la especie está relegada a las zonas menos contaminadas de dichos ríos, posiblemente porque la elevada contaminación hace inviable la supervivencia de la especie en la mayoría de su curso. Este aspecto por tanto podría confirmar que $P$. antipodarum tolera especialmente la contaminación relacionada con los vertidos de materia orgánica y la eutrofización, y menos otros tipos de contaminación como pueden ser los metales pesados y escorrentías ácidas procedentes de actividades mineras. Otro aspecto interesante es que la especie parece verse favorecida por ambientes fluviales con velocidades de corrientes no muy elevadas (Holomuzki y Biggs 2007; Álvarez-Cabria et al. 2011b), esto podría indicar como la regulación de caudales por medio de pequeñas presas (i.e. azudes) podría favorecer la aparición de hábitats adecuados para la especie. Por lo tanto, la eliminación de estas estructuras supondría un incremento en la velocidad de la corriente en esos tramos y tal vez una reducción en la densidad de la especie (o su imposibilidad para colonizar el ambiente).

Esta revisión muestra que probablemente $P$. antipodarum ha llegado a la península ibérica por la costa cantábrica y/o mediterránea y desde ahí se ha expandido hacia el interior, sin que aparentemente ningún clima y tipo de sustrato haya detenido su avance. A la vista de los resultados sí que podemos afirmar con elevada probabilidad que la especie se ha extendido en las últimas décadas por la península ibérica. Por el contrario, la información sobre los impactos de la especie en la estructura y funcionamiento de los diferentes ecosistemas acuáticos invadidos es muy escasa, aunque la información disponible en otros países hace pensar que su impacto es importante. Además, se hace necesario el estudio de los principales mecanismos de dispersión entre las cuencas fluviales de la península ibérica, en un intento de implementar medidas que puedan controlar y parar la aparente propagación de esta especie exótica.

\section{Agradecimientos}

La presente investigación ha sido financiada por los siguientes proyectos de investigación: Ministerio de Ciencia e Innovación (CGL2010-16388/BOS), Universidad de Alcalá (CCG2013/EXP054) y red REMEDINAL de la Comunidad de Madrid (S2009/AMB1783). Agradecer los comentarios de dos revisores anónimos y del editor para mejorar el contenido del estudio.

\section{Referencias}

Alonso, A. 2005. Valoración de la degradación ambiental y efectos ecotoxicológicos sobre la comunidad de macroinvertebrados bentónicos en la cabecera del río Henares. Tesis Doctoral, Universidad de Alcalá, Alcalá de Henares, España, 197 pp.

Alonso, A., Camargo, J.A. 2003. Short-term toxicity of ammonia, nitrite and nitrate to the aquatic snail Potamopyrgus antipodarum (Hydrobiidae, Mollusca). Bulletin of Environmental Contamination and Toxicology 70:1006-1012.

Alonso, A., Camargo, J.A. 2004. Sub-lethal responses of the aquatic snail Potamopyrgus antipodarum (Hydrobiidae, Mollusca) to unionized ammonia: a tolerant invading species. Fresenius Environmental Bulletin 13: $607-615$.

Alonso, A., Castro-Díez, P. 2008. What explains the invading success of the aquatic mud snail Potamopyrgus antipodarum (Hydrobiidae, Mollusca)? Hydrobiologia 614:107-116.

Alonso, A., Castro-Díez, P. 2012a. The exotic aquatic mud snail Potamopyrgus antipodarum (Hydrobiidae, Mollusca): State of the art of a worldwide invasion. Aquatic Sciences 74: 375-383

Alonso, A., Castro-Díez, P. 2012b. Tolerance to air exposure of the New Zealand mudsnail Potamopyrgus antipodarum (Hydrobiidae, Mollusca) as a prerequisite to survival in overland translocations. NeoBiota 14: 67-74.

Álvarez-Cabria, M., Barquín, J., Juanes, J.A. 2010. Spatial and seasonal variability of macroinvertebrate metrics: Do macroinvertebrate communities track river health? Ecological Indicators 10: 370-379.

Álvarez-Cabria, M., Barquín, J., Juanes, J.A. 2011a. Macroinvertebrate community dynamics in a temperate European Atlantic river. Do they conform to general ecological theory? Hydrobiologia 658: 277-291.

Álvarez-Cabria, M., Barquín, J., Juanes, J.A. 2011b. Microdistribution patters of macroinvertebrate communities upstream and downstream of organic effluents. Water Research 45: 1501-1511.

Arluziaga, I. 2002. Variación de la calidad de las aguas de los ríos guipuzcoanos al cabo de veinte años (1981-2001). Munibe 53: 39-56.

Baur, B., Schmidlin, S. 2007. Effects of invasive non-native species on the native biodiversity in the river Rhine. En: Netwing W (ed) Biological invasions. Ecological studies, vol 193. Springer, Heidelberg, Alemania. pp 257-273.

Bech-Taberner, M., Altamiras-Roset, J. 2003. Nuevas aportaciones al conocimiento de los moluscos actuales y del cuaternario en Extremadura: I. Malacofauna dulceacuícola. Revista de Estudios Extremeños 2: 837-870.

Castro-Díez, P., Valladares, F., Alonso, A. 2004. La creciente amenaza de las invasiones biológicas. Ecosistemas 13: 61-68.

Castro-Díez, P., Godoy, O., Alonso, A., Gallardo, A., Saldaña, A. 2014. What explains variation in the impacts of exotic plant invasions on the nitrogen cycle? A meta-analysis. Ecology Letters 17: 1-12.

Correia, M.J., Costa, J.L., Chainho, P., Félix, P.M., Chaves, M.L., Medeiros, J.P., Silva, G., Azeda, C., Tavares, P., Costa, A., Costa, A.M., Bernardo, J., Cabral, H.N., Costa, M.J., Cancela da Fonseca, L. 2012. Inter-annual variations of macrobenthic communities over three decades in a landlocked coastal lagoon (Santo André, SW Portugal). Estuarine, Coastal and Shelf Science 110: 168-175.

Costa, A.M., Cristo, M., Cancela da Fonseca, L. 2003. Annual cycle of the benthic community of a coastal lagoon: Lagoa de Melides (Grândola, SW Portugal). Revista de Biología 21: 71-89.

Crooks, J.A., Chang, A.L., Ruiz, G.M. 2011. Aquatic pollution increases the relative success of invasive species. Biological Invasions 13: 165-176.

de la Puente, M., Camargo, J.A. 2006. Los macroinvertebrados del bentos. En: Julio A. Camargo, J.A. (ed.), Ecología y Conservación del Río Henares y sus Tributarios, pp. 109-136. Ediciones CERSA, Madrid, España.

Dorgelo, J., Leonards, P.E.G. 2001. Relationship between C/N ratio of food types and growth rate in the snail Potamopyrgus jenkinsi (E. A. Smith). Journal of the North American Benthological Society 20: 60-67.

Duft, M., Schulte-Oehlmann, U., Tillman, M., Markert, B., Oehlmann, J. 2003. Toxicity of triphenyltin and tributyltin to the freshwater mud snail Potamopyrgus antipodarum in a new sediment biotest. Environmental Toxicology and Chemistry 22:145-152.

Gangloff, M.M. 1998. The New Zealand mud snail in Western North America. Aquatic Nuisance Species 2: 25-30. 
Gavira Romero, O., Robles Domínguez, E., Becerra Parra, M., Larraz Azcárate, M.L. 2009. Presencia de Acicula norrisi Gittenberger y Boeters, 1977 (Gastropoda: Aciculidae) en España. Iberus 27:73-76.

Gèrard, C., Blanc, A., Costil, K. 2003. Potamopyrgus antipodarum (Mollusca: Hydrobiidae) in continental aquatic gastropod communities: impact of salinity and trematode parasitism. Hydrobiologia 493:167-172

Gherardi, F. 2007. Biological invaders in inland waters: profiles, distribution, and threats. Springer, Dordrecht

Hall, R.O. Jr., Dybdahl, M.F., VanderLoop, M.C. 2006. Extremely high secondary production of introduced snails in rivers. Ecological Application 16:1121-1131.

Hall, R.O. Jr., Tank, J.L., Dybdahl, M.F. 2003. Exotic snails dominate nitrogen and carbon cycling in a highly productive stream. Frontiers in Ecology and Environment 1:407-411.

Holomuzki, J.R., Biggs, B.J.F. 2007. Physical microhabitat effects on 3-dimensional spatial variability of the hydrobiid snail, Potamopyrgus antipodarum. New Zealand Journal of Marine and Freshwater Research 41:357-367.

Hosea, R.C., Finlayson, B. 2005. Controlling the spread of New Zealand mud snails on wading gear. State of California, The Resources Agency. Administrative Report 2005-02

Ibáñez, M., Alonso, M.R. 1977. Geographical distribution of Potamopyrgus jenkinsi (Smith 1889) (Prosobranchia: Hydrobiidae) in Spain. Journal of Conchology 29: 141-146.

Jensen, A., Forbes, V.E., Parker, D. Jr. 2001. Variation in cadmium uptake, feeding rate, and life-history effects in the gastropod Potamopyrgus an tipodarum: linking toxicant effects on individuals to the population level. Environmental Toxicology and Chemistry 20:2503-2513.

Jiménez, M.L., Larraz, M.L. 1986. Estudio malacológico del río Arga (Navarra). Munibe (Ciencias Naturales) 38: 61-73

Jokela, J., Lively, C.M., Dybdahl, M.E., Fox, J.A. 1997. Evidence for a cost of sex in the freshwater snail Potamopyrgus antipodarum. Ecology 78:452-460.

Jones, J.P.G., Rasamy, J.R., Harvey, A., Toon, A., Oidtmann, B., Randrianarison, M.H., Raminosoa, N., Ravoahangimalala, O.R., 2009. The perfect invader: a parthenogenetic crayfish poses a new threat to Madagascar's freshwater biodiversity. Biological Invasions 11: 1475-1482.

Kolar, C.S., Lodge, D.M. 2001. Progress in invasion biology: predicting invaders. Trends in Ecology and Evolution 16: 199-204

Kumschick, S., Bacher, S., Dawson, W., Heikkilä, J., Sendek, A., Pluess, T., Robinson, T.B., Kühn, I. 2012. A conceptual framework for prioritization of invasive alien species for management according to their impact. $\mathrm{Ne}$ oBiota 15: 69-100.

Larraz, M.L., Equisoain, J.J. 1993. Moluscos terrestres y acuáticos de Navarra (Norte de la península ibérica). Serie Zoologica. Servicio de Publicaciones de la Universidad de Navarra.

Lewin, I., Smolinski, A. 2006. Rare and vulnerable species in the mollusc communities in the mining subsidence reservoirs of an industrial area (The Katowicka Upland, Upper Silesia, Southern Poland). Limnologica 36:181-191.

Loo, S.E., Keller, R.P., Leung, B. 2007. Freshwater invasions: using historical data to analyse spread. Diversity and Distributions 13: 23-32

Lowe, S., Browne, M., Boudjelas, S., De Poorter, M. 2000. 100 of the world's worst invasive alien species a selection from the global invasive species database. Published by the Invasive Species Specialist Group (ISSG) a specialist group of the Species Survival Commission (SSC) of the world conservation union (IUCN), p 12. First published as special liftout in Aliens Dec 12th 2000. (Updated and reprinted version)

Martínez-Ortí, A., Aparicio, M.T., Robles, F. 2004. La malacofauna de la Sierra de Alcaraz (Albacete, España). Iberus 22:9-17.

Mellado-Díaz, A. 2005. Ecología de las Comunidades de Macroinvertebrados de la Cuenca del Río Segura (SE de España). Tesis Doctoral, Universidad de Murcia, Murcia, España.

Mellado-Díaz, A., Suárez Alonso, M.L., Vidal-Abarca-Gutiérrez, M.R. 2008 Biological traits of stream macroinvertebrates from a semi-arid catchment: patterns along complex environmental gradients. Freshwater Biology 53: 1-21.
Múrria, C., Bonada, N., Prat, N. 2008. Effects of the invasive species Potamopyrgus antipodarum (Hydrobiidae, Mollusca) on community structure in a small Mediterranean stream. Fundamental and Applied Limnology 171/2: 131-143.

Netwing, W. 2007. Biological invasions: why it matters. En: Netwing, W. (ed) Biological invasions. Ecological studies, vol 193, pp 1-6. Springer, Heidelberg, Alemania.

Ortiz, J.D., Martí, E., Puig, A. 2005. Recovery of the macroinvertebrate community below a wastewater treatment plant input in a Mediterranean stream. Hydrobiologia 545: 289-302.

Ortiz, J.D., Puig, A. 2007. Point source effects on density, biomass and diversity of benthic macroinvertebrates in a Mediterranean stream. River Research and Applications 23: 155-170.

Oscoz, J., Tomás, P., Durán, C. 2010. Review and new records of non-indigenous freshwater invertebrates in the Ebro River basin (Northeast Spain). Aquatic Invasions 5: 263-284.

Pérez-Quintero, J.C. 2009. Freshwater molluscs from the biosphere reserve "Dehesas de Sierra Morena", SW Iberian Peninsula. Iberus 27:1-18

Pérez-Quintero, J.C. 2011. Freshwater mollusc biodiversity and conservation in two stressed Mediterranean basins. Limnologica 41: 201-212.

Rahel, F. J. 2007. Biogeographic barriers, connectivity and homogenization of freshwater faunas: it's a small world after all. Freshwater Biology 52:696-710.

Richards, D.C. 2002. The New Zealand mud snail invades the Western United States. Aquatic Nuisance Species 4:42-44.

Riley, L.A., Dybdahl, M.F., Hall, R.O. Jr. 2008. Invasive species impact: asymmetric interactions between invasive and endemic freshwater snails. Journal of the North American Benthological Society 27: 509-520

Rolán, E. 2004. Sobre una peculiar población de Potamopyrgus antipodarum en el tramo final del río Miño. Noticiario de la Sociedad Española de Malacología 42:50-53.

Sepulveda, A.J., Marczak, L.B. 2012. Active dispersal of an aquatic invader determined by resource and flow conditions. Biological Invasions 14: 1201-1209.

Serrano, L., Reina, M., Arechederrea, A, Casco, M.A., Toja, J. 2004. Limnological description of the Tarelo lagoon (SW Spain). Limnetica 23:1-10.

Simoes, M. 1988. Distribucion en Portugal de Potamopyrgus jenkinsi (Prosobranchia Hydrobiidae). Iberus 8:243-244.

Soler, J., Moreno, D., Araujo, R., Ramos, M.A. 2006. Diversidad y distribución de los moluscos de agua dulce en la comunidad de Madrid (España). Graellsia 62: 201-252.

Sousa, R., Antunes, C., Guilhermino, L. 2005. Molluscan fauna in the freshwater tidal area of the River Minho estuary, NW of Iberian Peninsula. International Journal of Limnology 41: 141-147.

Sousa, R., Antunes, C., Guilhermino, L. 2007. Species composition and monthly variation of the Molluscan fauna in the freshwater subtidal area of the River Minho estuary. Estuarine Coastal and Shelf Science 75: 90-100.

Svobodova, J., Douda, K., Stambergova, M., Picek, J., Vlach, P. 2012. The relationship between water quality and indigenous and alien crayfish distribution in the Czech Republic: patterns and conservation implications. Aquatic Conservation-Marine and Freshwater Ecosystems 22: 776-786.

Terhivuo, J., Saura, A. 2006. Dispersal and clonal diversity of North-European parthenogenetic earthworms. Biological Invasions 8: 1205-1218.

Vinson, M.R., Baker, M.A. 2008. Poor growth of rainbow trout fed New Zealand mud snails Potamopyrgus antipodarum. North American Journal of Fish Management 28: 701-709.

Werner, H., Boeters, H.D., Guenther, A. 1994. On the freshwater mollusc fauna in the Spanish province of Cuenca. Malakologische Abhandlungen (Dresden) 17:1-9.Winterbourn, M.J. 1973. A guide to the freshwater mollusca of New Zealand. Tuatara 20:141-159.

Winterbourn, M.J. 1970. The New Zealand species of Potamopyrgus (Gastropoda: Hydrobiidae). Malacologia 10:283-321.

Zaranko, D.T., Farara, D.G., Thompson, F.G. 1997. Another exotic mollusc in the Laurentian Great Lakes: the New Zealand native Potamopyrgus antipodarum (Gray 1843) (Gastropoda, Hydrobiidae). Canadian Journal of Fisheries and Aquatic Sciences 54: 809-814. 